\title{
Conservation of Mobile Terminals' Battery Life Using Dynamic Power Control Algorithm
}

\author{
Oyewobi S. S, Onwuka E. $\mathbf{N}^{*}$ \\ Department of Communication Engineering, School of Information and Communication Technology (SICT), \\ Federal University of Technology, Minna, Niger State, Nigeria
}

\begin{abstract}
Most of our activities today are tied around being able to make a call or connect to the Internet with our Mobile devices. However, to optimally utilize the network and deliver the needed satisfaction to the user the mobile device needs to be powered. Sadly most mobile devices are powered by energy-limited batteries, which impose a constraint on the mobile terminal optimal performance and on the network utility. As a result, special emphasis is now being laid on extending the battery life of mobile devices. In this work, we present a simple and effective power control algorithm, which conserves battery power by dynamically responding to real-time network conditions to adjust mobile terminal's transmit power. BER (bit error rate) in measurement reports obtained from signals received from the mobile terminal were used to estimate signal quality that helps to determine the network conditions and adjust mobile terminal's transmit power accordingly. Our algorithm was tested using simulation models, and our results were compared with the Stepwise algorithm, which is a popular power control algorithm in literature. A reasonable improvement was recorded for length of operation time and battery-energy saved for the mobile terminal over stepwise algorithm.
\end{abstract}

Keywords Power Control, Battery-Life, Bit Error Rate, Step Wise Algorithm, Transmit Power

\section{Introduction}

Wireless communications have grown rapidly over the last five years, and that growth is expected to continue[11, 14]. Besides GSM (Global System for Mobile Communications) and 3G (Third Generation) mobile communication systems, new communication technologies such as $4 \mathrm{G}$ (Fourth Generation) Networks are emerging[8]. The trends in technological development in the area of networking points to a future of untethered communication[15, 23]; the dream of ubiquitous communication - anywhere anytime with any device is already being achieved. The phrase 'anywhere and anytime' means the device will most likely be a wireless device $[11,15]$. Also the idea of "always best connected" (abc) for users implies the device should be power confident[15]. However, the limited energy stored in battery of mobile devices have always been a constraint to users while trying to derive maximum benefit from the teeming network resources[11, 15, 23]. Therefore, considering the great importance of mobile terminal both for today and in the future, efficient power management of a mobile terminal is critical and is subject to optimisation[15]. It was for this reasons that research works in the field of mobile devices' power management and power control began in the early 1990s[6]. A

* Corresponding author:

onwukaliz@futminna.edu.ng (Onwuka E. N)

Published online at http://journal.sapub.org/jwnc

Copyright (C) 2011 Scientific \& Academic Publishing. All Rights Reserved literature review of past research works in this field, showed the different approaches different researchers have adopted at trying to find a solution to the challenge; their major contributions; and the gap in knowledge.

Consequently, in trying to fill a part of this gap we present "conservation of mobile terminals' battery life using dynamic power control algorithm. The rest of this paper is organised as follows: Section 2 presents related works; Section 3 presents our dynamic power control algorithm, in Section 4 results are presented while Section 5 concludes the paper.

\section{Related Works}

As wireless communications industry is growing, users' demands for new enhanced features and long battery life are increasing. Hence, power has become the first class design constraint, when it comes to rich multimedia portable devices $[12,18]$. This section presents insight to previous works that have been carried out in this area, their contributions and their drawbacks.

Sklavos et al[20] titled their work, "Power Consumption in wireless network: Techniques \& Optimizations." In their attempt to provide solutions to the increasing energy demands, they proposed various power management techniques. Among them, the most well known is the voltage and frequency scaling technique. Their study showed that CPUs, memories and the device's display are the modules that 
consume most of the power resources. Their solution is a system-level based approach. According to[6], a system-level approach focuses on the power consumption of the CPUs, memories, buses or the display of the device. Since[20] suggests that the three most important contributors in power loss is CPU, the memory and the display, they proposed power management techniques that mainly involved these subsystems. For instance, in order to minimize the power loss due to CPU operation, they employed power efficient processors. According to them, memories' power loss can either be static or dynamic. In solving the problem they employed energy efficient memory schemes such as memory hierarchy, memory partitioning, and a new range of mobile/cellular memories such as cellularRAM and mobileRAM, which have been designed for wireless battery operating devices. While addressing Power Control Techniques for Displays in order to reduce the power consumed by the display they proposed the use of energy adaptive LCD systems. This technique suggests the trade off between quality and power consumption. That is, depending on the application requirements and users' demands, a reduction of brightness or size of the display, was also suggested for power efficiency. They claimed that this approach is further motivated by research results that showed that the window of focus, meaning the user's area of interest - utilizes only $60 \%$ of the total screen area. Based on these observations, techniques that modify the clarity of the image and colour depth of the non-active screen areas were proposed, while leaving the active screen area unchanged.

It could appear that[20] have solved all of the problems by tackling the three most power loss contributors as they claimed, but their solution is only a system based approach. According to research in the study of power consumption in portable phones, the RF subsystem rank second only to video and audio processing operations which they have been silent about[9], and this is the main focus of our work.

Abukmail et al[1] in their work "Energy Management for Mobile Devices through Computation Outsourcing within Pervasive Smart Spaces", explored the opportunity Pervasive Spaces could provide as supplemental energy sources. They utilize the nature of pervasive smart spaces to outsource computation that would normally be performed on a mobile device to a surrogate server within the smart space. The decision to outsource a computation according to them depends on whether its energy cost on the device is larger than the cost of communicating its data to the surrogate and receiving the results back. They proposed an approach by which the outsourcing decision is made at runtime, while the intelligence that makes that decision is inserted at compile-time as logic that modifies the application code. The merit of their approach as they claimed is that it is application-independent and requires minimal programmer energy awareness. Additionally, they implemented a runtime support in Linux to facilitate testing and experimenting with the client/server outsourcing approach. Their experimental validation and benchmarks they claimed, showed a significant energy saving on the mobile device, which they be- lieved validates their approach as a viable and novel approach to power saving and management for mobile devices.

First, their approach depends on a surrogate server for computation and energy saving through Computation Outsourcing within Pervasive Smart Spaces, this makes their system power management technique dependent, and nothing was said on power management for the surrogate, which means they assume that the surrogate will always be up or powered by some means which, they didn't mention. They claimed that their system makes communication a means of energy saving, but they didn't tell us who bears the cost of communication between the system and the surrogate. Their technique involves a lot of complex programming in java and $\mathrm{C}++$, and takes a lot of memory space and processor time, which consume reasonable amount of energy. They also claimed their outsourcing is real-time, but we know that this depends a lot on the processing power of the surrogate. Our design solves a whole lot of this problem by solving the problem of optimization within the system, and the network only.

Pushkar et al[16] in their survey which they captioned "Survey Report on Dynamic Power Management (DPM)" surveyed a series of works on dynamic power management and concluded that DPM can be applied to various levels in a system. To analyse a DPM based system, they adopted a bottom-up view. They first focused on how a power-manageable system component (e.g., chips) has been designed. Then they moved on to see how this DPM policy is applied to a functionally complete system, which binds all interacting components.

However, these simple minded policies may turn out to be inefficient. Firstly, they come with additional overhead since these policies have to be run on an operating system. Another weakness of the DPM is that based on its stochastic and predictive nature, policies are implemented on prediction of future events based on past history and or events resulting sometimes in over predictions and under predictions; consequently, a policy is likely to issue more shutdown commands and degrade performance. On the other hand, a policy can be conservative in power saving and issue fewer shutdown commands. While performance and accuracy improve, these policies consume more power.

Also policies are system, component and most time operation-specific, therefore specific policy has to be developed for every particular operation with its own assumptions, limitations and technicalities. However, our algorithm is simple and dynamic, taking into cognisance the dynamic nature of wireless network environment, this it achieves by measuring the quality (BER) of the received signal at the base station and adjusting the transmit power accordingly.

In Ref.[4] Ashay et al proposed a dynamic power control algorithms in their work "Algorithms for Transmission Power Control in Biomedical Wireless Sensor Networks." They proposed a feedback-based closed-loop algorithms for dynamically adjusting radio transmit power in body-worn devices, they evaluated the performance of their algorithm in terms of energy savings and reliability as the data periodicity 
and feedback time-scales vary with real life experimental set-up. Using experimental trace data from body worn devices, they first showed that the performance of dynamic power control is adversely affected at long data periods. Next for a given data period they showed that modifying the transmit power at too long time scales (around a minute) reduces the efficacy of dynamic power control, while too short a time-scale (few seconds or less) incurs a high feedback signalling overhead. They therefore advocate an intermediate range of time-scales (when permitted by the data periodicity), typically in the few tens of seconds, at which the control algorithms should adapt transmit power in order to achieve maximal energy savings in body-worn sensor devices used for medical monitoring.

While their work is good and recent, it only addressed two aspects of power control technique, which affect dynamic power control, and with particular focus on wireless sensor network; namely the periodicity of data transmissions and the frequency of transmit power updates. Albeit the performance of their algorithm was tested in terms of energy savings and reliability, energy saving was not their primary interest, their primary interest was to evaluate the effect of data periodicity and feedback time-scales on dynamic power control.

Though our work employs power control, our algorithm is simple and our focus is on conserving energy at the mobile terminal using a power control algorithm that takes into consideration the ever-changing nature of the mobile network environment. We have incorporated this into the algorithm by measuring two parameters; signal strength and signal quality at every measurement period.

Our technique is however related to that used in the Step-wise power control algorithm[7, 10]. Note that the base station controls the power output of the mobile terminal through the concept of Power Control. In the Step-wise Power Control a Table of GSM power levels is defined, and the base station controls the power of the mobile terminal by sending a GSM "power level" number to it. The mobile terminal then adjusts its power accordingly. In all cases the increment/decrement between the different power level numbers is $2 \mathrm{~dB}$. Measurement reports are sent in the Slow Associated Control Channel (SACCH) about once in 480 $\mathrm{ms}[7,10]$.

The Step-wise is good in the sense that it is dynamic and it is real-time. However, it has two major limitations which are complexity owing to the amount of parameters that needs to be predefined. The other problem is the usage of a fixed incremental/decremental value for all situations[15] i.e., it decrements or increments in steps of $2 \mathrm{~dB}$ to get to the desired power level. This stepwise approach slows down the response of the system to power control needs. For this reason, the algorithm does not conserve power enough. Our objective is to design an algorithm that will solve this problem by increasing the speed of response to power control needs of the system.

\section{Dynamic Power Control Algorithm}

The dynamic power control algorithm (DPCA) being proposed in this work evaluates and adjusts the transmitted power to the desired signal strength and quality by a power regulation process that regulates the transmitted power directly to the required optimum transmitted power.

\subsection{Simulation Environment}

The simulation model in this work is based on the real-life GSM system network and all design concepts were modelled according to the OPNET design procedures.

The project domain in our design comprised of;

1. Base station

2. Mobile terminal

Figure 1 is a typical view of our project domain.

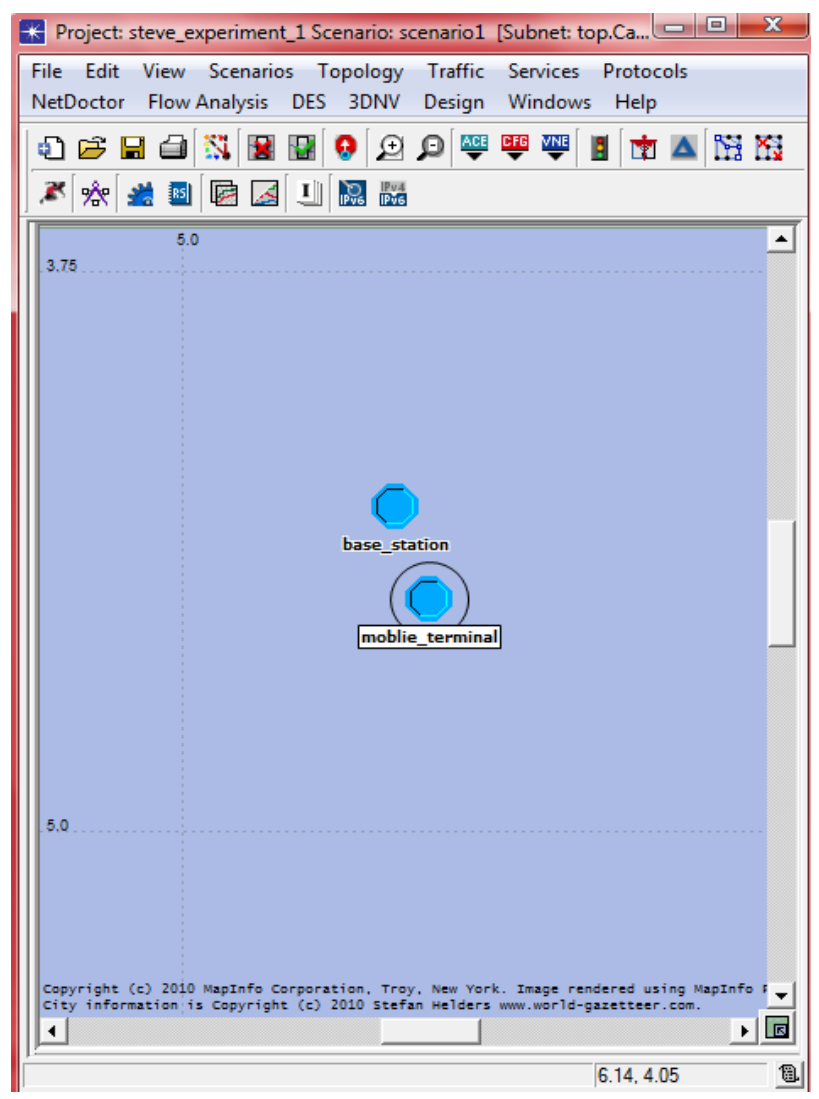

Figure 1. Project domain consisting of the base station and the mobile terminal

Our network topology was designed with the following parameters:

1. Total network coverage area $=10 \times 10 \mathrm{Km}$.

2. The mobile terminal was located at coordinates $(0.5$, $0.5)$ in $\mathrm{Km}$.

3. The base station was located at coordinates $(1.5,1.5)$ in $\mathrm{Km}$.

The process model in OPNET hierarchy is where all algorithms, protocols, and designs are coded into the OPNET modeler. It is in this module therefore that our algorithm and all calculations are coded into the simulator. The process model in our design consists of three statistical transmission diagram (STDs). It consists of an init state for the initialization and registration of statistical variables, a packet-wait 
state and a processor. After initialization at the init state, there is an unconditional transition to the packet-wait state, which serves as a buffer for packets before they are sent to the processor.

\subsection{Design Values Estimation}

The following parameters obtained from standard GSM network and Nokia mobile phone were used for calculation.

i. Maximum power transmitted for the mobile is set at 39 $\mathrm{dB}$ as specified by GSM 900. Note that GSM 900 specifies 5 classes of power[3, 10].

ii. Maximum battery capacity is set at $700 \mathrm{mAH}$, a typical value for Nokia mobile phone battery. Note that one Ampere-hour $(1 \mathrm{AH})$ is equal to a current of one ampere flowing for one hour. This means that if you have a two Ampere-hour battery, then it has the capacity to push a current of two Ampere for one hour or a current of one Ampere for two hours.

iii. A maximum voltage of $3.7 \mathrm{~V}$ was set, which is also typical of the model of the Nokia phone battery.

iv. $700 \mathrm{mAH} \times 3.7 \mathrm{~V}=2.59 \mathrm{WH}$. Approx. $3 \mathrm{WH}$

v. $3 \mathrm{~W} \times 60 \mathrm{~s}=180 \mathrm{Ws}$, this is the value of available battery power at the start of transmission.

vi. Available battery Power $=$ Maximum Capacity Power Drain value

\subsection{Simulation Parameter Considerations}

i. The Dynamic Power Control Algorithm takes into consideration both $\mathrm{Rx}_{\text {Qual }}$ (receive quality) and $\mathrm{Rx}_{\mathrm{Lev}}$ (receive level), which enable the MT's (mobile terminal) power to increase when there is low $\mathrm{Rx}_{\text {Qual }}$ and/or low $\mathrm{Rx}_{\mathrm{Lev}}$.

ii. If the received signal strength or quality $\left(\mathrm{Rx}_{\mathrm{Lev}}\right.$ or $\left.\mathrm{Rx}_{\mathrm{Qual}}\right)$ increases or decreases from the set threshold, the transmit power of MT decreases or increases respectively.

iii. If the $\mathrm{Rx}_{\mathrm{Lev}}$ or $\mathrm{Rx}_{\text {Qual }}$ is optimum, there will be no variation in output power[2].

iv. Instruction for the change in power for the regulation is given by:

$$
\begin{array}{r}
P u=\propto\left(\text { ssdes }- \text { rxlev }_{\text {filtered }}\right)+\beta(\text { qdes } d B- \\
\text { rxqualfiltered } d B(1)
\end{array}
$$

Where $\alpha$ and $\beta$ are the path loss respective quality compensation and $\mathrm{q}_{\mathrm{des}} \mathrm{dB}$ (desire quality) and $\mathrm{rx}_{\text {qualfiltered }} \mathrm{dB}$ (receive quality filtered) are the $\mathrm{q}_{\mathrm{des}}$ and $\mathrm{rx}_{\text {qual }}$ mapped to $\mathrm{C} / \mathrm{I}$ as in Table 1.

Table 1. desired/received signal quality

\begin{tabular}{|c|c|c|c|c|c|c|c|c|}
\hline $\mathrm{q}_{\text {des }}(\mathrm{dtqu})$ & 0 & 10 & 20 & 30 & 40 & 50 & 60 & 70 \\
\hline $\mathrm{Rx}_{\text {qual }}$ & 0 & 1 & 2 & 3 & 4 & 5 & 6 & 7 \\
\hline $\mathrm{C} / \mathrm{I}(\mathrm{dB})$ & 23 & 19 & 17 & 15 & 13 & 11 & 8 & 4 \\
\hline
\end{tabular}

1. The power level down regulation order is given by:

$$
M S_{\text {power }}=P_{\max }-2 P L,
$$

where $I N T$ truncates the power level to a higher level value and $P_{\max }$ corresponds to maximum power.

The purpose of the Bit Error Rate measurement update in
Table 2 is to serve the Dynamic Power Control algorithm with dynamic $\mathrm{q}_{\mathrm{des}}$ values that change automatically. These measurement reports give additional information about the current quality in the system.

Table 2. BER measurement updates

\begin{tabular}{|c|c|c|c|c|}
\hline $\mathrm{BER}(\%)$ & $<0.2$ & $0.21-0.4$ & $0.41-0.8$ & $0.81-1.6$ \\
\hline $\mathrm{Rx}_{\text {qual }}$ & 0 & 1 & 2 & 3 \\
\hline $\mathrm{C} / \mathrm{I}(\mathrm{dB})$ & 23 & 19 & 17 & 15 \\
\hline $\mathrm{BER}(\%)$ & $1.61-3.2$ & $0.321-6.4$ & $6.41-12.8$ & $>12.8$ \\
\hline $\mathrm{Rx}_{\text {qual }}$ & 4 & 5 & 6 & 7 \\
\hline $\mathrm{C} / \mathrm{I}(\mathrm{dB})$ & 13 & 11 & 8 & 4 \\
\hline
\end{tabular}

\subsection{Steps Towards Validating Experimental Hypothesis}

(i). Experiment 1: Set up experiment to study the effect of transmission between the MT and the base station without any form of power control.

$=>$ Note the time taken for mobile terminal battery to get completely depleted.

(ii). Experiment 2: Set up experiment to study the effect of transmission with dynamic Power Control Algorithm on the mobile terminal battery.

$=>$ Note the effect of algorithm on mobile terminal battery power.

Note time taken for mobile terminal battery to get completely depleted.

(iii). Experiment 3: Set up experiment to study the effect of transmission with step wise algorithm on the mobile terminal battery.

$=>$ Note the effect of the algorithm on the mobile terminal battery power.

Note time taken for mobile terminal battery to get completely depleted.

Each of the experiments 1, 2 and 3 was ran for three different network scenarios, which we termed: Good network condition (for BER $<0.2$ ), Moderate network condition (for BER between $1.61 \& 3.2$ ), and Bad network condition (for $\mathrm{BER}>12.8)$

\section{Results}

Figure 2 shows the effect of transmission between the mobile terminal and the base station without Power Control on the battery power of the mobile terminal. $180 \mathrm{Ws}$ of battery energy at the start of the experiment was completely used up after 23 minutes of a 1 hour transmission simulation time.

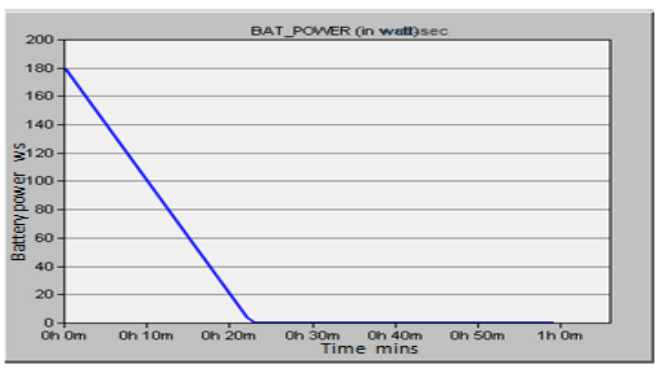

Figure 2. Effect of transmission without power control on MT's battery 


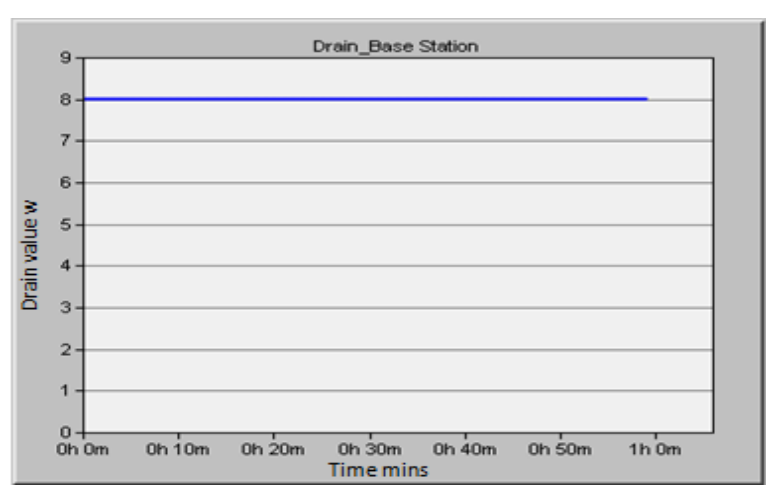

Figure 3. Effect of transmit Power regulation without power control

\section{STEP-WISE ALGORITHM}

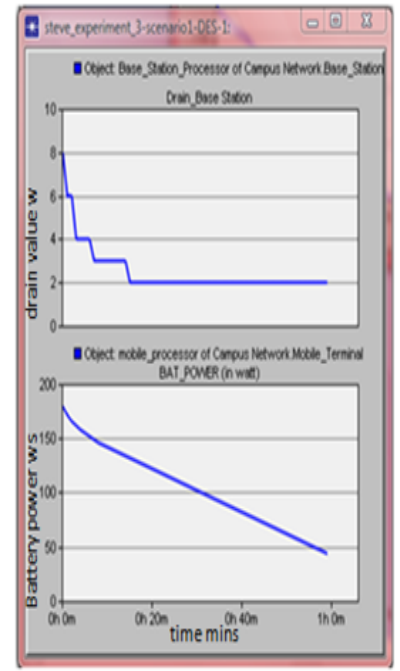

\section{DYNAMIC ALGORITHM}

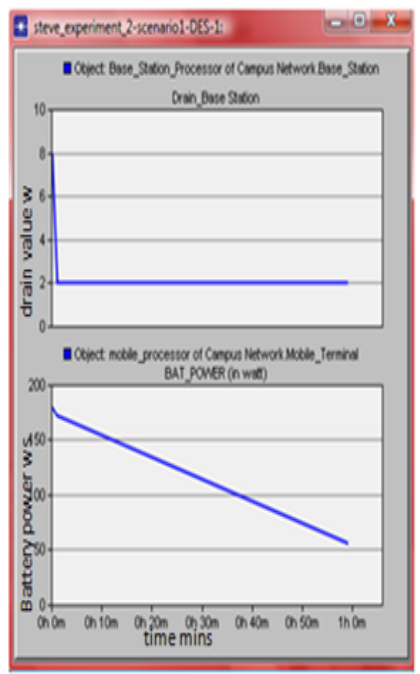

Figure 4. Power Controls and their effects on battery power of MT in good network condition

Figure 3 is the Power regulation process for the transmission between the MT and the base station without Power Control. The MT continuously transmitted at the default maximum transmit-power of $8 \mathrm{~W}$ because there was no Power Control.

Figure 4 shows the Power regulation processes and their effects on the Battery Power of the mobile terminal for both the stepwise and our dynamic power control algorithm in a Good Network Condition.

While the stepwise algorithm steps down transmit power from the default transmit power of $8 \mathrm{~W}$ to the optimum of $2 \mathrm{~W}$ in decremental fashion. Our dynamic Power Control algorithm having calculated $2 \mathrm{~W}$ to be optimal for the prevailing conditions in the network; regulated the transmit power directly from the default transmit power of $8 \mathrm{~W}$ to the optimum transmit power of $2 \mathrm{~W}$ in faster response time.

For stepwise algorithm, $136 \mathrm{Ws}$ of battery power out of the $180 \mathrm{Ws}$ available battery power was used up after 1 hour transmission; however in the case of our dynamic power algorithm $122 \mathrm{Ws}$ of battery power out of the $180 \mathrm{Ws}$ available battery power was used up after 1 hour of transmission. This shows about $8 \%$ saving in battery power due to the algorithm used.

Figure 5 shows results for Moderate Network Condition, the different power regulation processes and their effects on the battery of the mobile terminal. The stepwise algorithm regulated transmit power in its trademark decremental fashion, our dynamic power control algorithm had a faster response by regulating transmit power directly to the optimal transmit powers.

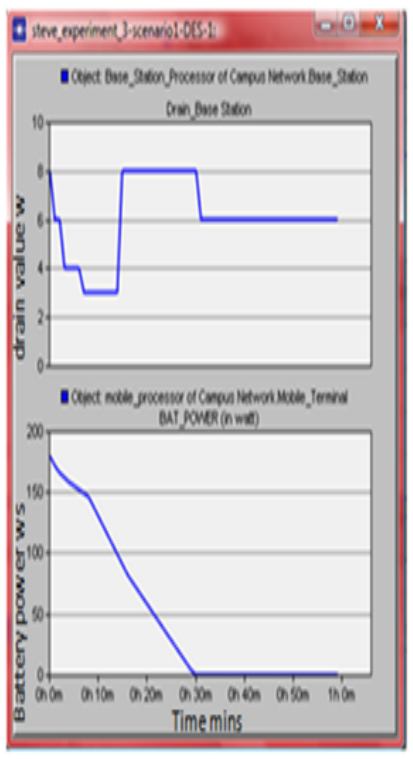

\section{STEP.WISE ALGORITHM}

\section{DYNAMIC ALGORITHM}

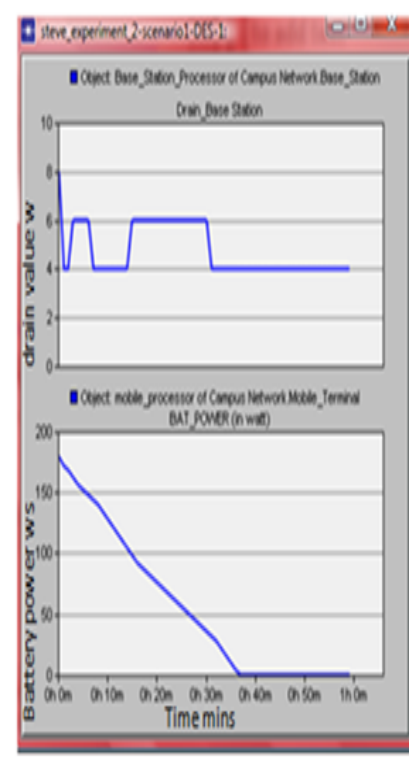

Figure 5. Power Control and their effects on battery power of mobile terminal in a moderate network condition

For stepwise algorithm, mobile terminal battery power was completely used up after 30 minutes of transmission. However our dynamic power algorithm extends transmission to 38 minutes before battery power was used up. This is about $27 \%$ of extended battery life due to the algorithm used.

For bad network condition, both algorithms responded similar to the case of transmission without power control shown in Figure 3, this happened because network condition was bad; therefore transmission was allowed to continue at peak power to be able to maintain good connection between the mobile terminal and the base station[5, 13].

\section{Conclusions}

This work focused on the need to fully optimise available battery power of a transmitting mobile terminal. It has been shown that signal transmission alone can consume up to one-third of a mobile phone's battery power; excluding that consumed by non-transmitting-energy-consuming components of the mobile phone. Efforts have been made by researchers in developing algorithms to control the transmit power of a mobile phone so that it does not transmit at unnecessarily high power. However, most of the algorithms in literature do not perform equally well due to their methods of operation. One such example is the Step-wise power control algorithm, which though regulates transmit power has a slow response and therefore performs poorly. Therefore we de- 
signed our dynamic power control algorithm, which has a faster response to transmit power control and as a result optimized the available battery power of the transmitting mobile terminal better when compared with the popular Stepwise algorithm.

\section{REFERENCES}

[1] Abukmail, A. A. and Helal, A. S. (2007).“Energy Management for Mobile Devices through Computation Outsourcing within Pervasive Smart Spaces".

[2] Amit, d., Sheel, S. (2002). Performance Optimization in GSM Networks through Dynamic Power Control.@ IEEE.

[3] Andrew, L. (2009). Extend your mobile phone's battery life. CNET.co.uk

[4] Ashay, D., Vidit, M., Shuo, X. (2009) Algorithms for Transmission Power Control in Wireless Sensor Networks. University of New South Wales Sydney, Australia.

[5] Basil, M. K., Muzhir, S., Josef, H., Rafa, E.A. (2008). Measuring the Optimal Transmission Power of GSM Cellular Network: A Case Study. Communications of the IBIMA

[6] Benini, L, De micheli, G (2000) .System-Level Power Optimization: Techniques and Tools. ACM Transactions on Design Automation of Electronic Systems, Vol. 5, No. 2, x.

[7] Croce, W. (2004). Cell Phones Demand Better Battery Life. Electronic Design Europe ,EEPN Microwaves \& RF Schematics

[8] Etoh, M. (2005). Trends in Mobile Multimedia and Networks. DoCoMo Communications Laboratories USA.

[9] Fredrik, H. (2005). "Improved power control for GSM/EDGE". masters thesis Lulea University of Technology Department of Computer Science and Electrical Engineering,GSM basics tutorial and overview. Radio-electrnnics.com

[10] Jones, C., Sivalingam, K., Agrawal,P., Chen, J., (2001). A Survey Of Energy Efficient Network Protocols For wireless Networks. Wireless Networks 7, 343-358, Kluwer Academic Publishers. Manufactured in The Netherlands.

[11] Lahiri, K. Z. Raghunathan, A. Y. Dey, S., Panigrahi, D.(2002). Battery-Driven System Design: A New Frontier in Low Power Design. Dept. of ECE, UC San Diego, La Jolla, CA,C \& C Research Labs, NEC USA, Princeton, NJ

[12] Mishra. (2004). Second Generation Network Planning and Optimisation (pp 6-10).

[13] Olwal, T., Wyk, B., Ntlatlapa, N., Djouani, K., Siarry, P., Hamam,Y.(2010). Dynamic Power Control for Wireless Backbone Mesh Networks: a Survey. Network Protocols and Algorithms ISSN 1943-3581 2010, Vol. 2, No. 1

[14] Onwuka, E.N., Oyewobi, S.S. (2011). "Implementation and Evaluation of the Effect of Dynamic Power Control on Operation-Time of a Mobile Terminal". Book of Proc., 2nd Biennial Engineering Conference. SEET, FUT, Minna, Nov 2010, pp 76-81.

[15] Pushkar, S and Chinta, V. (2009). Survey report on dynamic power management. University of Illinois, Chicago

[16] Sandip, H.C. (2007). System for improving overall battery life of a gsm communication device USPTO Applicaton \#: \#2007 (USPTO).

[17] Shun-Ren, Y (2005). Dynamic Power Saving Mechanism for 3 G UMTS System. Department of Computer Science, National Tsing Hua University, Hsinchu, Taiwan,

[18] Shun-Ren, Y., Yi-Bing L. (2008). Performance Analysis of UMTS Power Saving Mechanism. National chiao tung university taiwan R.O.C

[19] Sklavos, N., Touliou, K. (2007). Power Consumption in Wireless Networks: Techniques \& Optimizations. Region 8, EUROCON 2007.

[20] Walter, C. (2004). Cell Phones Demand Better Battery Life.[online] Available: www.planetee.com.

[21] Walter, j., Thomas, L., Dietmar S. (2004). Power Management for RF-Powered vs. Battery-Powered Devices. Scheiblhofer Infineon Technologies Austria AG Graz, Austria.

[22] Zheng, R., Jennifer, C.. Hou, L ., Ning, L.(2005). Power Management And Power Control In Wireless Networks. Department of Computer Science, University of Illinois at Urbana-Champaign, Urbana, IL 61801. 